G. SONG AND J. HUANG

KODAI MATH. J.

10 (1987), $42-48$

\title{
ON PSEUDO-PRIMALITY OF THE $n$-TH POWER OF PRIME ENTIRE FUNCTIONS
}

\author{
By GuOdong Song And Jue HuANG
}

\section{Introduction and main results.}

Let $g_{0}(z)$ be a transcendental entire function which is prime or pseudoprime. We pose the following question: are the functions $g_{0}(z)^{n}$ always pseudoprime for $n=2,3, \cdots$ ? The answer is affirmative if and only if $n$ is an odd number. That is to say that $g_{0}(z)^{n}$ is pseudo-prime if $n$ is odd; while for even number $n$, there exists a prime entire function $g_{0}(z)$ such that $g_{0}(z)^{n}$ is not pseudo-prime. This assertion is contained in the following two theorems.

THEOREM 1. Let $g_{0}(z)$ be a pseudo-prime entire function, and $n(\geqq 3)$ be an odd number. Then $F(z)=g_{0}(z)^{n}$ is also pseudo-prime.

THEOREM 2. The function

$$
F(z)=(\sin z) e^{\cos z}
$$

is prime.

Remark 1. If $F(z)$ is the function of the form (1), and $n$ is an even number, then $F(z)^{n}$ is not pseudo-prime, as is shown by the following factorization

$$
F(z)^{n}=\left(\sin ^{n} z\right) e^{n \cos z}=\left(\left(1-w^{2}\right)^{n / 2} e^{n w}\right) \cdot \cos z .
$$

Remark 2. The function $F(z)$ of the form (1) is also an example of prime periodic entire functions. In 1971, Gross [4] asked if there exist such functions. Later on, Ozawa [8,9], Baker \& Yang [2], Gross \& Yang [6] constructed various examples of such kind of entire functions. Our example here is a much simpler one.

From Theorem 2 and Remark 1, it is easy to derive the following

COROLlaRY. For any polynomial $P(z)$ of degree 2, there exists a prime entire function $g^{*}(z)$ such that $F(z)=P\left(g^{*}(z)\right)$ is not pseudo-prime.

The basic notions in the factorization theory of entire and meromorphic

Received April 7, 1986 
functions, such as prime, $E$-prime, pseudo-prime, etc., shall not be stated here. One may find the definitions of these notions in the references.

\section{Preliminary lemmas.}

In proving our theorems we shall need several known results.

LEMMA 1 (Hayman [7]). Let $f(z)$ be an entrre function. Then

$$
\sum_{a \neq \infty}\left(1-\frac{1}{v(a)}\right) \leqq 1
$$

where $v(a)$ stands for the least order of almost all a-pornts of $f(z)$. Especially, there is at most one complex number a such that $v(a) \geqq 3$.

LEMMA 2 (Edrei [3]). Let $g(z)$ be an entire function. If there exists an unbounded sequence $\left\{a_{n}\right\}$ such that almost all the roots of $g(z)=a_{n}(n=1,2, \cdots)$ lie on one straight line, then $g(z)$ is a polynomial of degree at most two.

LEMMA 3 (Baker \& Gross [1]). Let $h(z)$ be a periodic entire function of finite lower order, and $c$ be a non-zero constant. Then

is prime.

$$
H(z)=h(z)+c z
$$

LEMMA 4 (Gross [5]). All meromorphic solutions of the functional equation

$$
f(z)^{2}+g(z)^{2}=1
$$

are of the form

$$
f(z)=\frac{2 s(z)}{1+s(z)^{2}}, \quad g(z)=\frac{1-s(z)^{2}}{1+s(z)^{2}},
$$

where $s(z)$ is any meromorphic function. In particular, there is no non-constant $f(z)=z^{-n} f_{0}(z)$ where $n$ is a non-negative integer and $f_{0}(z)$ is a polynomial satisfying equation (2).

\section{Proof of theorem 1.}

Let $n=p_{1} \cdot p_{2} \cdots p_{k}$ with prime numbers $p_{j} \geqq 3, \jmath=1, \cdots, k$. If $g_{1}(z)=g_{0}(z)^{p_{1}}$ is proved to be pseudo-prime, so is $g_{2}(z)=g_{1}(z)^{p_{2}}=g_{0}(z)^{p_{1} p_{2}}$, and so on. Therefore, we may assume that $n=p$ is a prime number. Also, $g_{0}(z)$ may be assumed transcendental.

Suppose $F(z)=g_{0}(z)^{p}=f(g(z))$ with transcendental entire functions $f$ and $g$. By Lemma 1 , it is easily seen that among zeros of $f(w)$ there is at most one zero with order $q$ such that $(q, p)=1$. Hence 2 cases may occur. 
(a) $f(w)=\left(w-w_{0}\right)^{q} h(w)^{p}$ and $g(z)=w_{0}+s(z)^{p}$ with transcendental entire functions $h$ and $s$. Then

or

$$
g_{0}(z)^{p}=s(z)^{q p}\left(h\left(w_{0}+s(z)^{p}\right)\right)^{p}
$$

$$
g_{0}(z)=u s(z)^{q} h\left(w_{0}+s(z)^{p}\right) \quad\left(u^{p}=1\right),
$$

which gives a contradiction as $g_{0}(z)$ is assumed to be pseudo-prime.

(b) $f(w)=h(w)^{p}$ with a transcendental entire function $h$. Then $g_{0}(z)=$ $u h(g(z))$. Again a contradiction.

Now let $F=f(g)$ with $f$ being meromorphic (not entire) and $g$ entire, both transcendental. Then $f$ must have exactly one pole, $w_{0}$ say, which $g$ doesn't take. And we may write

$$
f(w)=\left(w-w_{0}\right)^{-k} f^{*}(w), \quad g(z)=w_{0}+e^{M(z)},
$$

where $k$ is a positive integer, $f^{*}$ is transcendental entire with $f^{*}\left(w_{0}\right) \neq 0$, and $M(z)$ is non-constant entire.

If $f^{*}(w)$ has no zeros, or each zero of $f^{*}$ is of order $m p$ with a positive integer $m$, then $f^{*}(w)=h(w)^{p}$ with a transcendental entire function $h$, which implies

or

$$
g_{0}(z)=u e^{-k M(z) / p} h\left(w_{0}+e^{M(z)}\right) \quad\left(u^{p}=1\right)
$$

$$
g_{0}(z)=\left(u w^{-k} h\left(w_{0}+w^{p}\right)\right) \circ e^{M(z) / p} .
$$

But this violates the pseudo-primality of $g_{0}(z)$.

If $f^{*}(w)$ has a zero, $w_{1}$ say $\left(w_{1} \neq w_{0}\right)$, of order $q$ with $(q, p)=1$ (By the same reasoning as in case(a), $f^{*}$ has at most one such zero). Then $g(z)$ must be of the form

$$
g(z)=w_{1}+s(z)^{p}
$$

with an entire function $s$. But this is impossible, since the entire function $g(z)$, which has a Picard exceptional value $w_{0}$, can not have any completely ramified values.

The proof of theorem 1 is completed.

\section{Proof of theorem 2 .}

Let $F(z)=f(g(z))$ with non-linear entire functions $f$ and $g$. We discuss two cases.

Case (a). $f$ has infinitely many zeros. Then by lemma $2, g(z)$ must be a polynomial of degree 2 . Hence, $F(z)$ may be expressed by

$$
F(z)=f_{1}\left((z-c)^{2}\right)
$$

with an entire function $f_{1}$ and a constant $c$. This yields 


$$
\sin (z+c) e^{\cos (z+c)}=\sin (-z+c) e^{\cos (-z+c)} .
$$

But the above equality can not hold, as is shown by substituting some special values of $z$.

Case (b). $f$ has only finitely many zeros. Then we may write

$$
f(w)=Q(w) e^{L(w)}
$$

with a non-constant polynomial $Q$ and entire function $L$. Thus

$$
Q(g(z)) e^{L(g(z))}=(\sin z) e^{\cos z} .
$$

Since $F(z)=(\sin z) e^{\cos z}$ is of infinite order and its convergent exponent of zeros, denoted by $\rho^{*}(F)$, is one, if $L$ is a constant, then $Q$ must have exactly one (simple) zero, i.e. $Q$ is linear, which is out of our consideration. Therefore, $L$ must be non-constant.

By considering the growth of functions in both sides of (3), we see that the order of $g(z)$

and

$$
\rho(g) \leqq \rho(L(g))=\rho(\cos z)=1
$$

$$
\rho(g)=\rho(Q(g)) \geqq \rho^{*}(Q(g))=\rho^{*}(\sin z)=1
$$

So that $\rho(g)=1$.

Putting

$$
s(z)=\frac{Q(g(z))}{\sin z} \exp (\cos z-L(g(z))),
$$

we have $\rho(s) \leqq 1$, which implies

$$
\cos z-L(g(z))=a z+b, \quad a \text { and } b \text { are constants. }
$$

If $a \neq 0$, then by lemma $3, L(g(z))=\cos z-a z-b$ is prime, so that $L$ is linear. And we may write

$$
g(z)=c_{1} \cos z+c_{2} z+b_{1}
$$

where $c_{1}, c_{2}$ and $b_{1}$ are constants with $c_{1} c_{2} \neq 0$. On the other hand

$$
Q(g(z))=(\sin z) e^{a z+b} .
$$

Therefore, substituting $z=2 n \pi$ into both sides of (4), we see that the right side of (4) is 0 , while the left side tends to $\infty$, which is a contradiction. identity

If $a=0$, then $L(g(z))=\cos z-b$ and $Q(g(z))=b_{2} \sin z$, and we obtain an

$$
Q^{*}(g(z))^{2}+L^{*}(g(z))^{2} \equiv 1 \quad \text { or } \quad Q^{*}(w)^{2}+L^{*}(w)^{2} \equiv 1
$$


with a polynomial $Q^{*}$ and an entire function $L^{*}$ for every $w \in \boldsymbol{C}$, which violates lemma 4.

Up to now we have proved that $F(z)$ is $E$-prime.

Now, let $F=f(g)$ with meromorphic functions $f$ and $g$ ( $f$ is not entire), we discuss three cases.

Case (i). $f$ is transcendental. Then $g$ must be entire, and we have

$$
f(w)=\left(w-w_{0}\right)^{-n} f_{1}(w), \quad g(z)=w_{0}+e^{M(z)},
$$

where $f_{1}(w)$ is a transcendental entire function with $f_{1}\left(w_{0}\right) \neq 0, n$ is a positive integer, and $M(z)$ is a non-constant entire function. We derive

$$
\begin{aligned}
F(z) & =(\sin z) e^{\cos z}=e^{-n M(z)} f_{1}\left(w_{0}+e^{M(z)}\right) \\
& =\left[e^{-n w} f_{1}\left(w_{0}+e^{w}\right)\right] \cdot M(z)
\end{aligned}
$$

Since $F(z)$ is $E$-prime, $M(z)$ must be linear, and we may write

$$
F(z)=(\sin z) e^{\cos z}=e^{-n a z} f^{*}\left(e^{a z}\right),
$$

where $f^{*}$ is trancendental entire.

By the same argument as in case (a), we conclude that $f^{*}$ has only finitely many zeros. Then we may write

$$
f^{*}(w)=P(w) e^{N(w)}
$$

with a non-constant polynomial $P$ and entire function $N$. We obtain

$$
(\sin z) e^{\cos z}=e^{-a n z} P\left(e^{a z}\right) \exp \left(N\left(e^{a z}\right)\right)
$$

Putting

$$
T(z)=\frac{P\left(e^{a z}\right)}{\sin z}=\exp \left(\cos z+n a z-N\left(e^{a z}\right)\right) .
$$

Obviously, $\rho(T) \leqq 1$. Hence

$$
\cos z+n a z-N\left(e^{a z}\right)=A z+B, A \text { and } B \text { are constants. }
$$

If $A \neq n a$, then $N$ is linear (by using lemma 3 ), and we would get

$$
e^{a z}=A_{1} \cos z+A_{2} z+B_{1},
$$

which is apparently impossible.

If $A=n a$, then $N\left(e^{a z}\right)=\cos z-B$ and $P\left(e^{a z}\right)=B_{2}(\sin z) e^{n a z}$, and we would derive an identity 


$$
P^{*}\left(e^{a z}\right)^{2} e^{-2 n a z}+N^{*}\left(e^{a z}\right)^{2} \equiv 1 \quad \text { or } \quad \frac{P^{*}(w)^{2}}{w^{2 n}}+N^{*}(w)^{2} \equiv 1
$$

with a polynomial $P^{*}$ and $a$ positive integer $n$. This again violates lemma 4 .

Case (ii). $f$ is rational and $g$ entire. Then we obtain (5) and (6) with $f_{1}$ (and $f^{*}$ ) being a polynomial. And we may deduce that $M(z)$ is linear. But in this case the function in the right side of (6) would be of finite order, which is also a contradiction.

Case (iii). $f$ is rational and $g$ meromorphic (not entire). Let $x_{0}$ be a pole of $f$, then $g(z)$ doesn't assume $x_{0}$, so that

is entire. Denoting

$$
g_{1}(z)=\frac{1}{g(z)-x_{0}}
$$

$$
R(w)=f\left(\frac{1}{w}+x_{0}\right)
$$

we get a factorization $F=R \circ g_{1}$ which is equivalent to $F=f \circ g$. Then this case reduces to case (ii).

The proof is thus completed.

\section{Final remark.}

We propose the following questions:

(1) Does there exist an entire function $g_{0}(z)$ which is prime and of finite order such that $g_{0}(z)^{2}$ is not pseudo-prime?

(2) Let $P(z)$ be a polynomial of degree $\geqq 3$ which has no right factor of the form $(z-a)^{2}+b$ with constants $a, b$ and let $g_{0}(z)$ be a pseudo-prime entire function. Can we conclude that the function $F(z)=P\left(g_{0}(z)\right)$ is also pseudoprime?

\section{REFERENCES}

[1] BaKer, I. N. AND Gross, F., Further results on factorization of entire functions, Proc. Symp. Pure Math. (Amer. Math. Soc., Providence, R. I.) II (1968), 30-35.

[2] BAKER, I.N. AND YANG, C.C., An infinite-order periodic entire function which is prime, In: Proceedings of a Conference on Complex Analysis (Lexington 1976) pp. 7-10, Lecture Notes in Mathematics, 599, Berlin-Heidelberg-New York: Springer 1977.

[3] EDREI, A., A meromorphic function with three radially distributed values, Trans. Amer. Math. Soc., 78 (1955), 276-293.

[4] Gross, F., Factorization of entire function which are periodic mod $g$, Indian J. Pure Appl. Math., 2 (1971), 561-571.

[5] Gross, F., On the equation $f^{n}+g^{n}=1$, Bull. Amer. Math. Soc., 72 (1966), 86-88.

[6] Gross, F. ANd Yang, C.C., On prime periodic entire functions, Math. Z., 174 
(1980), 43-48.

[7] Hayman, W.K., Meromorphic functions, Clarendon Press, Oxford, 1964.

[8] OzAwA, M., Factorization of entire functions, Tohoku Math. J., 27 (1975), 321-336.

[9] OzAWA, M., On the existence of prime periodic entire functions, Kodai Math. Sem. Rep., 29 (1978), 308-321.

Department of Mathematics EAst China NoRmal University ShaNghaI, China
Department of Mathematics,

Huainan Mining College

Anhui, China 